\title{
Pancreatic sphincterotomy improves pain symptoms due to branch-duct intrapapillary mucinous neoplasia without worrisome features: a multicenter study
}

\section{(ㄷ)(i)}

\author{
Authors \\ Marc Giovannini ${ }^{3}$, Marc Barthet ${ }^{1}$ \\ Institutions \\ 1 Aix-Marseille University, APHM, Hôpital Nord, \\ Department of Gastroenterology, Marseille, France \\ 2 APHP, Hôpital Beaujon, Department of Pancreatology, \\ Clichy-la-Garenne, Paris, France \\ 3 Institut Paoli Calmette, UEMCO, Marseille, France \\ 4 CHU de Purpan, Department of Gastroenterology, \\ Toulouse, France
}

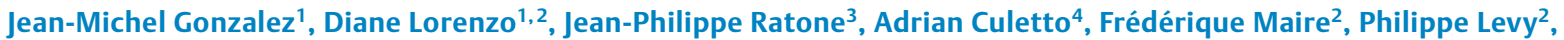

submitted 10.8.2018

accepted after revision 27.12.2018

\author{
Bibliography \\ DOI https://doi.org/10.1055/a-0841-3385 | \\ Endoscopy International Open 2019; 07: E1130-E1134 \\ (c) Georg Thieme Verlag KG Stuttgart · New York \\ eISSN 2196-9736
}

\section{Corresponding author}

Dr Jean-Michel Gonzalez, MD, APHM, Aix-Marseille

University, Department of Gastroenterology, Hôpital Nord, Chemin des Bourrelys, 13015 Marseille, France

Fax: +33-4-91968737

jean-michel.gonzalez@ap-hm.fr
ABSTRACT

Introduction Branch duct intraductal papillary mucinous neoplasms (BD-IPMNs) require follow-up to detect worrisome features (WF). Data are missing about endoscopic pancreatic sphincterotomy (PS) for symptomatic IPMN.

Patients and methods This was a retrospective multicenter study in four expert centers. Patients treated with endoscopic PS for symptomatic (painful) BD-IPMN without WF were included. Age, sex, follow-up time, characteristics of IPMNs and endoscopic retrograde cholangiopancreatographies (ERCPs), and indications for surgery were recorded. Results In total, 21 patients were included (median age 68 years, range 45-87 years). The median number of cysts was 2 (range $1-10$ ), located in the head (59\%), body/tail (17\%), or multifocal (24\%). ERCP including PS was completed in all of the cases, with biliary sphincterotomy in 33\%. Clinical efficacy after one session was $81 \%$ (17/21). Among the failures, one had a second successful PS and three were operated. The final efficacy was $86 \%(18 / 21)$. Seven patients were operated after a mean of 19 months: four for WF, three for pain. The histopathology showed four low grade dysplasia, one high grade dysplasia, and two no dysplasia. No adenocarcinoma occurred during a follow-up of 99 months (range 14-276 months).

Conclusions Endoscopic PS for symptomatic IPMN without WF is effective in more than $80 \%$ of cases, without increasing the risk for adenocarcinoma.

\section{Introduction}

Intraductal papillary mucinous neoplasms (IPMNs) require a close clinical and radiological follow-up to detect worrisome features (WF) early, with the risk of dysplasia or adenocarcinoma occurrence. The frequency of malignancy in a surgical series of resected side branch-duct IPMN (BD-IPMN) or in the case of EUS-follow-up is indeed about $20-25 \%$ [1,2]. On the other hand, symptoms such as pancreas related abdominal pain (PRAP) or recurrent acute pancreatitis (RAP) may occur with a rate varying from $12 \%$ to $67 \%$ of $B D-I P M N$ s in the largest published surgical series [3-5]. However, they are not always asso- ciated with WF in MRI or EUS [6] or with evolution towards malignancy [7]. The occurrence of malignancy in patients experiencing such symptoms is still a matter of debate despite surgical series, which suggests an increased risk even in BD-IPMN [8, 9]. The 2006 and 2012 international consensus [10, 11] included recurrent pancreatic pain in the indications for surgery for BD-IPMN. Nevertheless, the scientific evidence for such a recommendation could be controversial with regard to the risk linked to pancreatic surgery, particularly pancreatic duodenectomy.

The physiopathology of pancreatic symptoms in the case of BD-IPMN seems to be related to main pancreatic duct (MPD) 
occlusion by mucin plugs, secreted and coming from the cysts [12]. For this reason, it has been postulated that endoscopic treatment including pancreatic sphincterotomy might be helpful for managing mucinous obstructions. By enlarging the papilla's orifice, we aimed to suppress or at least significantly decrease the episodes of pancreatic pain. To our knowledge, there are few data about the efficacy of endoscopic sphincterotomy (ES) to manage symptomatic IPMN, apart from jaundice, and without WF. The current literature is composed of three small series including a total of six patients [13-15]. This could be of interest to avoid or to postpone a potential surgical resection.

For this reason, we conducted a multicenter retrospective study to evaluate the efficacy of PS, including a long-term follow-up of these patients to document their evolution towards dysplasia or adenocarcinoma.

\section{Patients and methods}

This was a retrospective multicenter observational study conducted from January 2005 to December 2016 in four tertiary centers. Each center is considered to be an expert center in the management of IPMN with a large volume of patients. A detailed survey was sent to them in order to record all of their patients treated with endoscopic pancreatic sphincterotomy for painful IPMN without WF. All diagnoses of IPMN had been confirmed in each center for each patient by the association of MRI and EUS showing a communicating cyst.

\section{Population}

More specifically, the inclusion criteria were: (i) patients older than 18 years old with BD-IPMN; (ii) follow-up for more than 6 months for IPMN without WF, solid mass or indication for surgery; (iii) suffering from either recurrent pancreatitis or postprandial abdominal pain (typical characteristics and no associated functional disease), or both; and (iv) treated for this indication by endoscopic pancreatic sphincterotomy. All of the patients had signed an informed consent with regard to the endoscopic retrograde cholangiopancreatography (ERCP) procedure before the procedure was performed.

For each patient, age, sex, medical history, time of follow-up (before and after ES), characteristics of IPMN (location on the pancreatic ducts, number and diameter of the cysts, WF) and of ERCP procedures, clinical success, evolution, and need/indication for surgery were recorded.

\section{Endoscopic procedures}

Endoscopic treatment was indicated based on the severity of symptoms with the absence of surgical indications. All of the procedures have been performed on patients under general anesthesia, their position depending on the operator's preference. Prophylaxis by NSAIDs was administered according to the team's protocol and current recommendations at the time. Pancreatic ES had to be performed in all of the cases, associated with a biliary sphincterotomy in the case of biliary tree dilation, biological hepatic test abnormality, and cholangitis, or in order to facilitate access to the pancreatic duct. The decision over placing a prophylactic plastic stent was left to the discretion of the endoscopist.

\section{Aims and end points}

The primary end point was to assess the efficacy of ES for improving the symptoms of pain related to IPMN. Clinical efficacy was defined as the resolution of PPAP or RAP (total efficacy), or a decrease of more than $50 \%$ of the frequency of the symptoms (partial efficacy), which was calculated based on the number of attacks per year. The follow-up period was at least 12 months and was stopped in the case of surgery (total efficacy), or a decrease of more than $50 \%$ of the frequency of the symptoms (partial efficacy), which was calculated based on the number of attacks per year. The follow-up period was at least 12 months and was stopped in the case of surgery.

The secondary end points were to document the characteristics of these IPMNs and the endoscopic treatment, the need for a second ES, the occurrence of WF or malignancy during follow-up, the need for surgery, and its indications.

\section{Results}

The files of 27 patients who underwent ES for symptomatic IPMN without WF or surgical indications between January 2008 and June 2016 were considered. Six were excluded, four because of a lack of data (lost in follow-up), one because it was a pseudocyst with chronic pancreatitis, and one that already had an adenocarcinoma. Finally, 21 patients were included in the study and their data analyzed.

\section{Characteristics of the patients and IPMNs}

These data are summarized in $>$ Table 1.

There were 10 men and 11 women in the study, with a median age of 68 years (range $45-87$ years). Their symptoms were PPAP in six patients (29\%) and RAP in the 19 others (90\%), five having had severe acute pancreatitis, and four patients having both RAP and PPAP. The median number of attacks was 3 per year (range 2-12). The median delay between initial diagnosis of IPMN and ES was 24 months (range 1-192 months). The patients were being followed by MRI in $81 \%$ of the cases, EUS in $95 \%$, CT scan in $36 \%$ or by alternating MRI and EUS in $45 \%$ of them.

The IPMNs involved the side branches of the pancreatic duct in $71 \%$ of the cases, and in $29 \%$ were associated with a Wirsung duct always measuring less than $7 \mathrm{~mm}$. The median number of cysts was 2 (range $1-10$ ), the largest one measuring a median of $10.5 \mathrm{~mm}$ (range $5-25 \mathrm{~mm}$ ). In $59 \%$ of patients, they were located in the head of the pancreas, in $17 \%$ of patients they were in the body or the tail, and they were multifocal with more than one location in $24 \%$ of the patients. No patient had worrisome features at the time of management.

\section{Endoscopic sphincterotomy: efficacy and characteristics}

An ERCP including the pancreatic ES was successfully performed in $100 \%$ of the cases. Total efficacy as defined was achieved in $15 / 21$ patients (71\%) and partial efficacy was 
- Table 1 Characteristics of patients including demography, symptoms and initial follow-up, and features of the IPMNs.

\begin{tabular}{|c|c|}
\hline \multicolumn{2}{|l|}{ Characteristic } \\
\hline Mean age (range), years & $66(45-87)$ \\
\hline \multicolumn{2}{|l|}{ Sex, n (\%) } \\
\hline - Male & $10 ; 48 \%$ \\
\hline - Female & $11 ; 52 \%$ \\
\hline \multicolumn{2}{|l|}{ Symptoms, n (\%) } \\
\hline - Post-prandial pain & $6 ; 29 \%$ \\
\hline - Recurrent pancreatitis & $19 ; 90 \%$ \\
\hline - Severe AP & 5 \\
\hline Median delay (DG/ES) (range), months & $24(1-192)$ \\
\hline \multicolumn{2}{|l|}{ Initial follow-up, \% } \\
\hline - MRI & $81 \%$ \\
\hline - EUS & $95 \%$ \\
\hline - CTscan & $36 \%$ \\
\hline - Alternating (MRI/EUS) & $45 \%$ \\
\hline \multicolumn{2}{|l|}{ Type of IPMN, \% } \\
\hline - Main pancreatic duct & $0 \%$ \\
\hline - Side branches & $71 \%$ \\
\hline - Mixed & $29 \%(\mathrm{MPD}<7 \mathrm{~mm})$ \\
\hline Median no.(range) of cysts & $2(1-10)$ \\
\hline Maximum diameter (range), mm & $12.7(5-25)$ \\
\hline \multicolumn{2}{|l|}{ Cyst location, \% } \\
\hline - Head & $59 \%$ \\
\hline - Body/tail & $17 \%$ \\
\hline - Multifocal & $24 \%$ \\
\hline \multicolumn{2}{|c|}{$\begin{array}{l}\text { AP, acute pancreatitis; DG/ES, diagnosis to endoscopic sphincterotomy; MRI, } \\
\text { magnetic resonance imaging; EUS, endoscopic ultrasound; CT, computed } \\
\text { tomography; IPMN, intrapapillary mucinous neoplasia; MPD, main pancre- } \\
\text { atic duct. }\end{array}$} \\
\hline
\end{tabular}

achieved in 2/21 (10\%). In total, the clinical efficacy rate of ES after one session was $81 \%(17 / 21)$. In the subgroup of patients with RAP or both $(n=19)$, the efficacy was $90 \%(17 / 19)$, against $50 \%$ in the two patients with PPAP.

Regarding the characteristics of the endoscopy, five patients had a pancreas divisum requiring a sphincterotomy of the accessory papilla, and a pre-cut was performed in two cases for difficult cannulation. A biliary ES was also performed in $33 \%$ of the cases, mostly for an associated biliary duct dilatation with biological cholestasis. A prophylaxis with NSAIDs was applied in only $38 \%$ of the patients, especially in the more recent ones, and a plastic stent was left in place at the end of the procedure in $33 \%$ of the cases. There were four post-ERC pancreatitis, none being severe.
Among the patients with primary clinical success $(n=17)$, one had a late recurrence after 41 months and underwent a second ES session with final success, whereas four have been operated (two for the initial pain despite the ES, two for WF occurrence); $19 \%(4 / 21)$ of the patients failed after the first attempt of endoscopic treatment. Among them, one had a second procedure including an enlargement ES for stricture, with final success, whereas three patients finally went for surgery. There were no statistically significant differences in the two groups in terms of age, sex, mean follow-up time, and characteristics of IPMN.

The median time of follow-up after ES was 41 months (range 12-167 months) and the median total follow-up was 74 months (range 12-282 months). The overall final efficacy, after one single endoscopic session including ES or after an additional one (stricture), was $86 \%(18 / 21)$. The results are summarized in the flow chart ( $\triangleright$ Fig. 1 ).

\section{Surgical outcomes}

In total, seven patients were operated after a median of 15 months of follow-up, $24 \%$ in the group of patients with successful ERCP and in $75 \%$ in the case of failure. The indications for surgery were the initial abdominal pain symptoms in three patients, in two cases, in spite of efficacy of the ES, this decision being made because pain was a worrisome feature for the team; and the appearance of WF in the post-ES follow-up for four patients.

Histopathological analysis of the specimens showed low grade dysplasia in four cases, high grade dysplasia in one case, and there was no dysplasia or metaplasia in two cases, both being surgery indicated for pain. Regarding the 14 patients who have not been operated, none evolved towards adenocarcinoma during the time of follow-up.

\section{Discussion}

Symptomatic IPMN with pancreatic symptoms including RAP have been described and may occur in about one-third of patients with BD-IPMNs $[16,17]$. In the recent international consensus [11], pain symptoms related to IPMN have been confirmed as indications for pancreatic surgical resection, consistent with the previous consensus. The rational for such an invasive approach was the risk of evolution towards malignancy. Indeed, classically, those patients with symptomatic IPMNs, even without any worrisome features on radiology (MRI or EUS), should undergo a pancreatectomy at least involving the part of the pancreas containing the largest side branch (SB) cyst.

However, there is very little evidence in the literature to support these recommendations, reported in two surgical series $[8,9]$. Consequently, one could consider there are some "nuances" to add to that statement. First, among the symptoms related to BD-IPMNs, only jaundice has clearly been linked to a higher risk for malignancy [18-20]. Second, some studies have suggested that, in the case of multifocal cysts, even in the case of resection of the main cyst, the efficacy on symptoms is questionable. Third, it seemed to us that such a severe and invasive surgery has to be really discussed and that ERCP may have a 


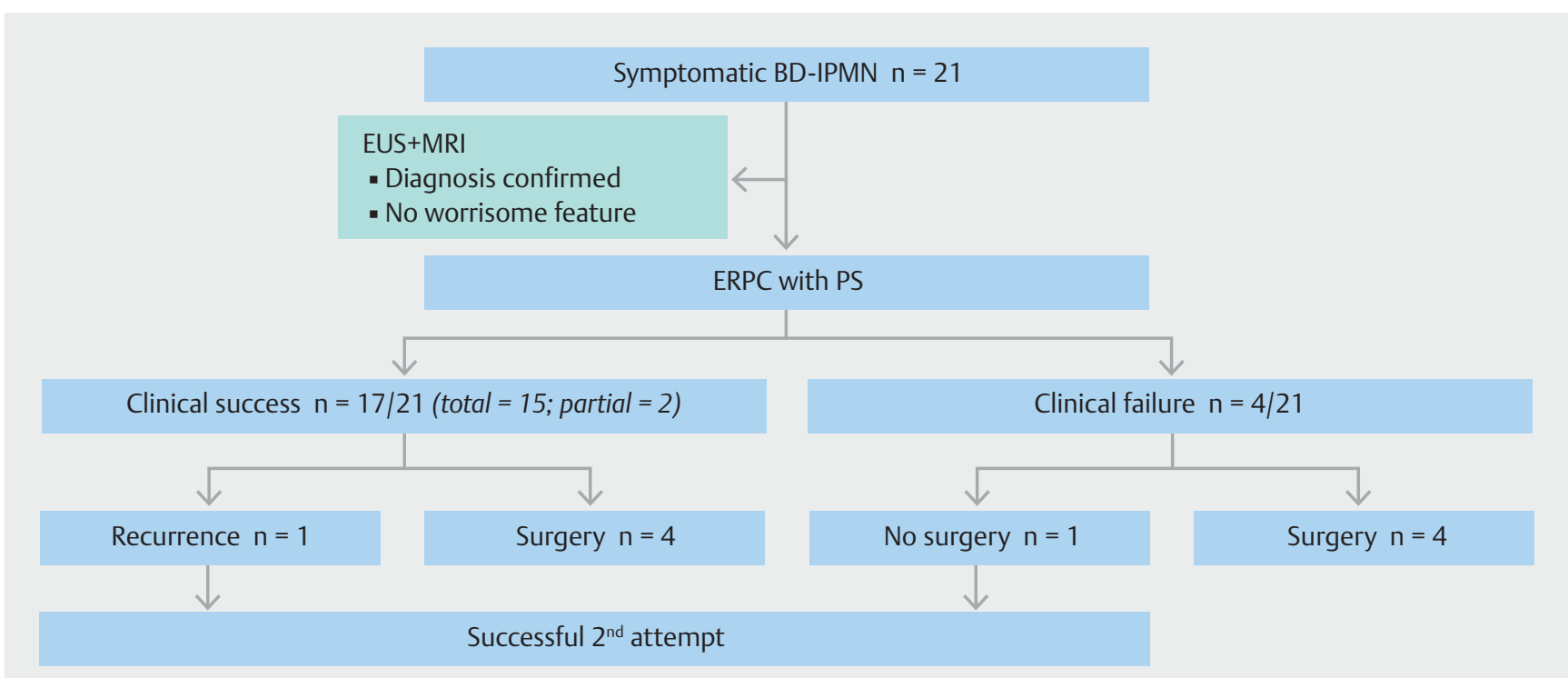

- Fig. 1 Flow chart summarizing the outcomes of patients in terms of response to pancreatic sphincterotomy as well as evolution during follow-up.

place to control the symptoms before surgery, in the case of absence of worrisome features.

Consequently, in the present multicenter study, we aimed to clearly assess for the first time the efficacy of ERCP with PS in the management of IPMNs with pancreatic symptoms without worrisome features. Our second objective was to answer the question about the risk of malignancy after a long-term follow-up in such symptomatic patients.

First, the efficacy rate of ES looks high, since it reached $81 \%$ after one single session, and $86 \%$ after a second session, usually indicated for a stenosis of the papillary orifice. Moreover, the procedures were safe, since there was no severe post-ERCP pancreatitis, even if NSAIDs were not systematically administered before the procedure. Also, interestingly, only one patient had a recurrence due to a benign pancreatic stricture managed endoscopically, and none have been operated for symptom recurrence, after a mean follow-up of almost 5 years. Clearly, none of the patients had main duct involvement (diameter $>10 \mathrm{~mm}$ ) or any worrisome feature, which would have indicated a surgical approach, consistent with the international guidelines. These results bring new and important information about the benefits of endoscopic treatment in the case of side branch IPMN with pancreatic symptoms, since there are no data in the current literature.

With regard to the evolution of these patients and the need for surgery, it is important to note that, in our series, no patients developed adenocarcinoma during a total follow-up of 103 months. Moreover, the indications for surgery were always the appearance of worrisome features (mural node or abrupt size increase) during the EUS follow-up, apart from one that was indicated for failure of endoscopic management. All of these patients $(n=5)$ were effectively found with dysplasia on specimen analysis. On the other hand, focusing on the two patients who had undergone pancreatectomy for their initial symptoms, despite the efficacy of the ES, they were operated erroneously since there was neither dysplasia nor metaplasia on histological analysis of their specimens.

In conclusion, ES for symptomatic IPMN without WF is a valuable therapeutic option, which is safe and completely effective in $70 \%$ of cases. It may allow unnecessary or early pancreatectomy to be avoided in patients who will not eventually have dysplasia or risk of malignancy. A symptomatic IPMN without jaundice does not seem to be a valuable indication for surgery, since no patients developed cancer after a follow-up greater than 5 years.

\section{Competing interests}

Dr. M. Barthet is a consultant for Boston Scientific. None of the other authors has a conflict of interest to disclose linked to this publication.

\section{References}

[1] Fernández-del Castillo C, Adsay NV. Intraductal papillary mucinous neoplasms of the pancreas. Gastroenterology 2010; 139: $708-713$, 713.e1-2

[2] Sawai $Y$, Yamao K, Bhatia $V$ et al. Development of pancreatic cancers during long-term follow-up of side-branch intraductal papillary mucinous neoplasms. Endoscopy 2010; 42: 1077-1084

[3] Venkatesh PGK, Navaneethan U, Vege SS. Intraductal papillary mucinous neoplasm and acute pancreatitis. J Clin Gastroenterol 2011; 2045: $755-758$

[4] D'Haese JG, Hartel M, Demir IE et al. Pain sensation in pancreatic diseases is not uniform: the different facets of pancreatic pain. World J Gastroenterol 2014; 20: 9154-9161 
[5] Jang JW, Kim M-H, Jeong SU et al. Clinical characteristics of intraductal papillary mucinous neoplasm manifesting as acute pancreatitis or acute recurrent pancreatitis. J Gastroenterol Hepatol 2013; 2028: $731-738$

[6] Warndorf M, Hu H, Papachristou G et al. Intraductal papillary mucinous neoplasm causing recurrent acute pancreatitis, necrotizing pancreatitis, and multifocal adenocarcinoma. Gastrointest Endosc 2014; 80: 1181 - 1182 discussion 1182

[7] Pelletier A-L, Hammel P, Rebours V et al. Acute pancreatitis in patients operated on for intraductal papillary mucinous neoplasms of the pancreas: frequency, severity, and clinicopathologic correlations. Pancreas 2010; 39: 658-661

[8] Morales-Oyarvide V, Mino-Kenudson M, Ferrone CR et al. Acute pancreatitis in intraductal papillary mucinous neoplasms: A common predictor of malignant intestinal subtype. Surgery 2015; 158: 12191225

[9] Tsutsumi K, Ohtsuka T, Oda Y et al. A history of acute pancreatitis in intraductal papillary mucinous neoplasms of the pancreas is a potential predictive factor for malignant papillary subtype. Pancreatology 2010; 10: 707 - 712

[10] Tanaka M, Chari S, Adsay V et al. International consensus guidelines for management of intraductal papillary mucinous neoplasms and mucinous cystic neoplasms of the pancreas. Pancreatology 2006; 6: $17-32$

[11] Tanaka M, Fernández-del Castillo C, Adsay V et al. International consensus guidelines 2012 for the management of IPMN and MCN of the pancreas. Pancreatology 2012; 12: $183-197$
[12] Asari S, Matsumoto I, Toyama H et al. Repeating regional acute pancreatitis in the head of the pancreas caused by intraductal papillary mucinous neoplasms in the tail: report of a case. Surg Today 2012; 42: $398-402$

[13] Oh YS, Dua K. Pancreatic sphincterotomy for pancreatitis associated with main duct intraductal papillary mucinous neoplasm. Endoscopy 2011; 43: (Suppl. 02): UCTN E75

[14] Elton E, Howell DA, Parsons WG et al. Endoscopic pancreatic sphincterotomy: indications, outcome, and a safe stentless technique. Gastrointest Endosc 1998; 47: 240-249

[15] Nocente R, Silveri NG, Gasbarrini A et al. An apparent idiopathic case of relapsing acute pancreatitis. Hepatogastroenterology 2001; 48: $572-573$

[16] Ringold DA, Shroff P, Sikka SK et al. Pancreatitis is frequent among patients with side-branch intraductal papillary mucinous neoplasia diagnosed by EUS. Gastrointest Endosc 2009; 70: 488 - 494

[17] Gourgiotis S, Ridolfini MP, Germanos S. Intraductal papillary mucinous neoplasms of the pancreas. Eur J Surg Oncol 2007; 33: 678-684

[18] Schmidt CM, White PB, Waters JA et al. Intraductal papillary mucinous neoplasms: predictors of malignant and invasive pathology. Ann Surg 2007; 246: 644-651 discussion 651-654

[19] Rodriguez JR, Salvia R, Crippa S et al. Branch-duct intraductal papillary mucinous neoplasms: observations in 145 patients who underwent resection. Gastroenterology 2007; 133: 72 - 79 quiz 309-310

[20] Moris M, Raimondo M, Woodward TA et al. Risk factors for malignant progression of intraductal papillary mucinous neoplasms. Dig Liver Dis 2015; 47: 495- 501 